\title{
A New Approach to Estimating the Path Loss in Underground Wireless Sensor Networks
}

\author{
Ali M. Sadeghioon, David N. Chapman *, Nicole Metje (1) and Carl J. Anthony \\ School of Engineering, University of Birmingham, Edgbaston, Birmingham, B15 2TT, UK; \\ a.m.sadeghioon@bham.ac.uk (A.M.S.); n.metje@bham.ac.uk (N.M.); c.j.anthony@bham.ac.uk (C.J.A.) \\ * Correspondence: d.n.chapman@bham.ac.uk; Tel.: +44-0121-414-5150
}

Received: 5 July 2017; Accepted: 4 August 2017; Published: 17 August 2017

\begin{abstract}
Unlike terrestrial Wireless Sensor Networks (WSNs), communication between buried nodes in WUSNs happens through the ground. Due to the complexity of soil, accurate estimation of the underground signal attenuation is challenging. Existing path loss models mainly rely on semi-empirical and empirical mixing models for calculating the dielectric properties of the soil. In this paper, two existing models for estimating the path loss in soil (i.e., the CRIM-Fresnel and Modified-Friis models) are compared with measurements obtained at three locations. In addition, an improved method is proposed for estimating the path loss based on a new approach for calculating the dielectric properties of soil from Time Domain Reflectometry (TDR) measurements. The proposed approach calculates the complex permittivity values from TDR waveform based on a new modified method and subsequently use them as inputs into the Modified-Friis model. The results from the field trials were compared with the proposed method and the existing models. The results of this comparison showed that the proposed estimation technique provides a better estimation of Radio Frequency (RF) attenuation than the existing models. It also eliminates the need to take samples back to the laboratory by providing in situ calculation of attenuation based on TDR.
\end{abstract}

Keywords: Wireless Underground Sensor Networks (WUSN); Time Domain Reflectometry (TDR); Path Loss; RF attenuation

\section{Introduction}

The design of the topology and deployment density of Wireless Sensor Networks (WSNs) are highly affected by the signal path loss estimation for each system in its intended environment. Wireless Underground Sensor Networks (WUSNs), as a subcategory of WSNs, have a variety of applications in precision agriculture, buried infrastructure monitoring (i.e., pipeline monitoring) and mine monitoring [1,2]. However, the environments that WUSNs operate in are drastically different from those for common terrestrial WSNs. These differences impose design and operational limitations on the WUSNs. The lack of available power and large path loss (caused by the poor radio frequency transmission through soil) impose boundaries on routing and topology of the networks, and are the main challenges for WUSNs [3].

Signal transmission in a WUSN can be divided into three main categories of Underground to Underground (UG2UG) and Underground to Aboveground (UG2AG and AG2UG). From a system viewpoint, Aboveground to Aboveground (AG2AG) may also be important for ultimately transmitting any information to the end user, but this is not part of the current assessment. Figure 1 shows a schematic of a typical WUSN used for pipeline monitoring. 


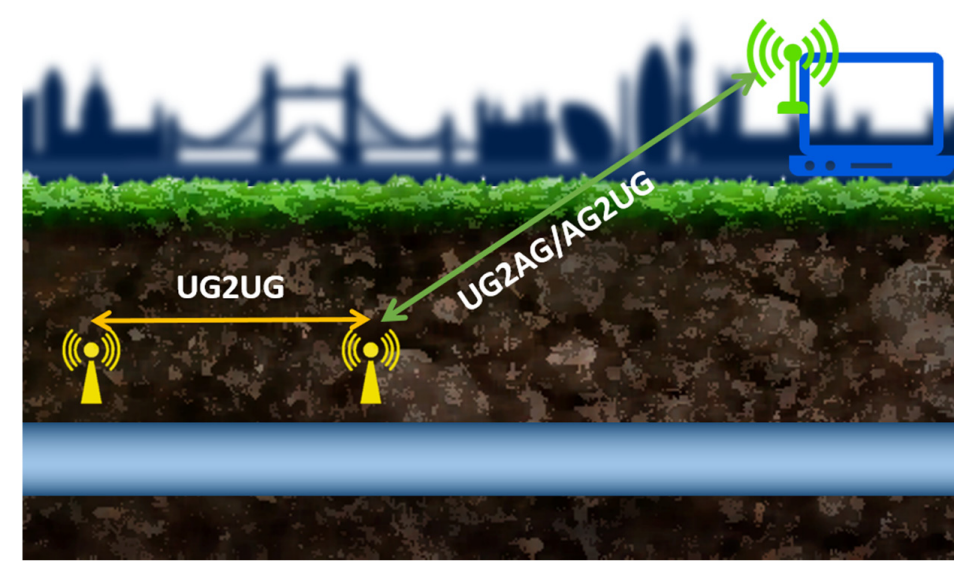

Figure 1. Schematic of a typical WUSN for pipeline monitoring.

UG2UG communications in WUSNs can be further categorized, based on their depth, into topsoil communications (0-30 cm depth) and subsoil communications ( $>30 \mathrm{~cm}$ depth). This classification aims to separate the effect of the soil-air boundary on the attenuation of the signals (i.e., reflections). The majority of buried infrastructure (e.g., water pipes) is placed in the subsoil region [4,5] and therefore the focus of this paper is on underground to underground wireless communication in this region.

While the attenuation of Electromagnetic (EM) signals in air is well understood and extensive research exists [6-8] on the path loss models for terrestrial WSN in various applications, the complex and dynamic nature of the soil have made it difficult to accurately estimate the attenuation of EM signals in soil. Many of the properties of soil, such as water content, composition, and density, greatly affect the attenuation of EM signals by changing the dielectric properties of soil [3]. In addition, these properties are not constant and can vary due to environmental factors (i.e., water infiltration due to rain). The path loss of the signals in the soil where the WUSN nodes are deployed should be accurately estimated in order to design an efficient and reliable WUSN. Moreover, the range of variations in the path loss due to environmental/seasonal changes should also be considered in the design of the WUSNs. An environment with a higher path loss can dictate the spatial density of deployment of the underground nodes to ensure reliable connectivity.

Soil can be defined as a complex mixed dielectric medium, which consists of air, water (bound and free) and bulk soil [9]. The composition of the soil and its water content are the two factors that are mainly used in empirical models for the estimation of the dielectric properties of soil [10]. Dielectric properties of soil can also be estimated based on Time Domain Reflectometry (TDR) and Vector Network Analyser (VNA) measurements [11,12].

The existing research [5,13-15] only reports on the performance of one model and/or one location, thereby making a more comprehensive comparison of the models/locations impossible. To the best knowledge of the authors, this paper, for the first time, compares the accuracy of common models for the estimation of RF attenuation with experimental measurements of attenuation in soil that have been undertaken at three separate field trial locations each having different soil compositions and conditions. This allows the models to be directly compared with each other at each location. In addition to the existing model approaches, a new approach for estimating the RF attenuation in soil is also investigated that proposes the use of permittivity values extracted from TDR measurements rather than mixing models [10]. The TDR extracted permittivity values are then applied to the Modified-Friis model to give an approach that does not require laboratory analysis of the soil at the location of the WUSN. This alternative approach is also then compared to the existing approaches and the RF attenuation measurements obtained from field trials. 


\section{RF Signals in Soil}

\subsection{Dielectric Properties of Soil}

Underground path loss estimation models commonly use complex dielectric permittivity, electrical conductivity and magnetic permeability of soil, which are the key dielectrics parameters, in their estimation calculations.

Dielectric permittivity is a complex value, which signifies the ability of a dielectric medium (i.e., soil) to permit the transmission of an electric field. The complex permittivity is defined by Equation (1) $[11,16]$.

$$
\varepsilon^{*}=\varepsilon^{\prime}-j \varepsilon^{\prime \prime}
$$

where $\varepsilon^{*}$ is the complex dielectric permittivity, $\varepsilon^{\prime}$ is the real part of complex permittivity (storage of energy), $\varepsilon^{\prime \prime}$ is the imaginary part of complex permittivity (relaxation and dispersive losses) and $j=\sqrt{-1}$. The permittivity of a medium is commonly described by the relative permittivity, $\varepsilon_{r}$, which is the ratio of the real part of the permittivity to the permittivity of free space $[10,11]$. The permittivity of the soil can be affected by many factors. However, the main parameters affecting the permittivity are the water content, the frequency of the signal, the soil composition and the soil conductivity. Unfortunately, the complex permittivity of soil cannot be easily measured. Therefore, estimating the permittivity of soil has been the topic of numerous research in the literature, and various methods have been proposed to model the permittivity of the soil based on other, more easily measurable parameters (e.g., composition and water content) [16-19]. These models are often known as mixing models. The existing estimation models for the attenuation of electromagnetic signals in soil rely heavily on the accuracy of the specific mixing model used for estimating the complex permittivity of soil.

The static electrical conductivity of soil can be described as the ability of the material to conduct electrical currents [11]. The imaginary part of the complex permittivity is directly affected by the value of the conductivity, which in turn affects the attenuation of electromagnetic signals in soil [20]. The electrical conductivity of soil can be easily measured both in situ and in the laboratory via a variety of techniques (e.g., TDR) [11].

The magnetic permeability of soil is commonly expressed as the relative magnetic permeability, which is defined as the ratio between the magnetic permeability of soil and free space [11]. For most common types of soil (i.e., non-iron oxide rich soils) the relative magnetic permeability of soil can be assumed to be $1[21,22]$.

\subsection{Propagation Models}

Accurately estimating the signal attenuation in soil can benefit a variety of fields where WUSNs are required. Therefore, multiple empirical and semi-empirical models exist in the literature, which aim to predict the attenuation of electromagnetic signals in soil. The two main models in the literature are the CRIM-Fresnel model and the Modified-Friis model [23-25]. Both of these models are based on the "link budget" formula, which was proposed by Akyildiz and Stuntebeck [3] to act as a framework for EM wave propagations models in soil. This formula adds a correction factor to the well-known Friis transmission equations in free space [26] to account for the extra losses in soil. The link budget formula is given by Equation (2).

$$
P_{r}=P_{t}+G_{r}+G_{t}-L_{0}-L_{m}
$$

where $L_{m}$ is the path loss in soil (medium) due to material absorption $(\mathrm{dB}), L_{0}$ is the path loss in free space $(\mathrm{dB}), G_{t}$ and $G_{r}$ are the transmitter and receiver antenna gains $(\mathrm{dB})$ and $P_{t}$ and $P_{r}$ are the transmitter and receiver powers $(\mathrm{dB})$.

Based on the Modified-Friis model, the total path loss caused by the medium, $L_{m}$, (i.e., soil) consists of losses caused by the change in the wavelength, and the attenuation losses caused by the 
material absorption [24]. Therefore, based on this model, the total losses of an EM signal in soil, $L_{p}$, including losses in free space, is given by Equation (3).

$$
L_{p}=6.4+20 \log (d)+20 \log (\beta)+8.68 \alpha d
$$

where $d$ is the distance between the transmitter and receiver and $\alpha$ and $\beta$ are given by Equations (4) and (5).

$$
\begin{aligned}
& \propto=\omega \sqrt{\frac{\mu \varepsilon^{\prime}}{2}\left[\sqrt{1+\left(\frac{\varepsilon^{\prime \prime}}{\varepsilon^{\prime}}\right)^{2}}-1\right]} \\
& \beta=\omega \sqrt{\frac{\mu \varepsilon^{\prime}}{2}\left[\sqrt{1+\left(\frac{\varepsilon^{\prime \prime}}{\varepsilon^{\prime}}\right)^{2}}+1\right]}
\end{aligned}
$$

where $\omega$ is the angular frequency $(\omega=2 \pi f)$ and $\mu$ is the relative magnetic permeability; and where the real and imaginary parts of the complex permittivity are estimated using the Peplinski dielectric mixing formula [17,18].

The CRIM-Fresnel model proposed by Bogena et al. [23] is a semi empirical model based on the Complex Refractive Index Model (CRIM). This model is also based on the link budget formula presented in Equation (2). However, unlike the Modified-Friis model, the CRIM-Fresnel model also considers losses due to reflection in the calculations of total attenuation, $A_{t o t}$ in soil $[15,21,23]$. Another difference between these two models is that the CRIM-Fresnel model uses the CRIM mixing model for estimating the complex permittivity of soil. The total attenuation loss, $\mathrm{A}_{t o t}$, based on the CRIM-Fresnel model, is given by Equation (6).

$$
A_{t o t}=\alpha_{c} d+R_{c}
$$

where $\alpha_{c}$ is the attenuation due to material absorption [23,27] and $R_{c}$ is the attenuation due to reflection [23]. Bogena et al. [23] claim that their proposed model has a better performance at estimating the attenuation in soil compared to the Modified-Friis due to the lack of a large supporting database for the Peplinski mixing model used by the Modified-Friis. However, the CRIM-Fresnel model itself was developed based on a very limited soil types and conditions. The soil used in the research by Bogena et al. [23] was stated to be "Sieved Sand", without providing further information on the type and particle size of the sand that was used; furthermore, it has not been validated by field trials [23].

A common drawback of both of these models is that their accuracy is largely dependent on the accuracy of the mixing model used. In addition, both of the mixing models used in the CRIM-Fresnel and Modified-Friis models require a sample of the soil to be analysed in the laboratory to accurately measure the parameters required for the mixing models. This makes them unsuitable for scenarios where estimation of attenuation of RF signals is required in situ (e.g., prior to geophysical surveys such as Ground Penetrating Radar (GPR) for utility location). In addition to these drawbacks, there is a large discrepancy between the estimated attenuation of EM signals determined from these models. The following section describes the methodology used for the field trials and the proposed method utilising in situ TDR measurements to determine the EM properties of the soil.

\section{TDR and Field Trials}

\subsection{Proposed Path Loss Estimation Method}

As mentioned in Section 2.1, the value of the complex permittivity of the soil significantly affects the attenuation of EM signals. Therefore, the accuracy of the estimation models for EM attenuation in soil is highly dependent on the accuracy of the permittivity values used for the calculations. An alternative method to the mixing models normally used for obtaining the complex values of permittivity is to extract the complex permittivity from TDR measurements [12]. This method is based 
on estimating the effective frequency of the TDR signal in the soil, which is the frequency that contains the majority of the energy of the signal [11], by calculating the rise time of the signal at the end of the TDR probe (Figure 2) [12]. The rise time of the signal is commonly calculated based on the $10 \%$ to $90 \%$ increase in the reflection coefficient at the end of the probe [11].

In practice, multiple reflections at the end of the probe can make it difficult to choose the top line (horizontal asymptote) for determining the rise time [11,28]. Sadeghioon [10] proposed that the steady state value, which is commonly used for calculations of Bulk Electrical Conductivity (BEC), is used for the value of the top line of the TDR waveform. This method eliminates inaccuracies caused by the multiple reflections of the waveform at the end of the probe and provides a more robust method for calculating the rise time. Figure 2 illustrates the proposed method for calculating the rise time of a waveform when there are multiple end reflections with rise time, $t_{r}$, and travel time, $t_{1}$, of the signal indicated.

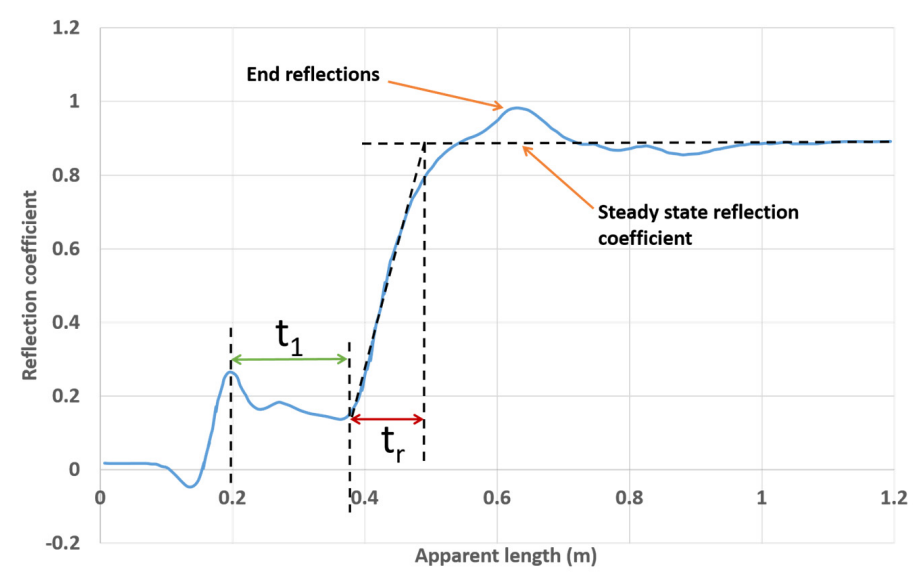

Figure 2. The proposed method of calculating the rise time in TDR waveforms with end reflections [10].

As can be seen from Figure 2, the end reflection in the TDR waveform in this figure would have resulted in longer rise time based on the conventional method, which would have adversely affected the accuracy of the calculated complex permittivity.

Following the calculation of the complex permittivity based on this alternative method (Figure 2), the permittivity is then used as an input to the Modified-Friis model to estimate the attenuation, instead of using Peplinski's mixing model for the permittivity values, thus eliminating potential inaccuracies associated with this mixing model. In addition, as TDR measurements can be taken in situ, it overcomes the previously mentioned limitations of the mixing models by providing an estimation of complex permittivity without the need for laboratory tests. The ability of this alternative approach for predicting RF attenuation in soil is tested by comparing the results to the standard models and, importantly, by comparing all the model results with attenuation data obtained from the field trials.

\subsection{Trial Setup}

Due to the challenges of conducting robust field trials, very few trials have been carried out investigating RF communication through soil $[4,16]$. During this research, the attenuation of RF signals in soil were measured at three separate locations (labelled A, B, C in the following sections) to provide a varied set of data for testing the performance of the models. The locations of the trials were selected to have different soil types and conditions. In addition, all of the locations had a soft surface (i.e., grass) in order to ease the process of digging and reinstating the ground, thus facilitating the implementation of the trials. The trial consisted of first excavating vertical holes in the ground with approximate diameters of 300-400 $\mathrm{mm}$, depth of $500 \mathrm{~mm}$ and spacing of 500-600 mm, created using hydraulic post hole borers and/or vacuum excavation techniques. The depth of these holes was selected to avoid reflections from the surface [9]. Although 2.4 GHz is a common frequency band used in terrestrial 
wireless sensor networks, it is not suitable for environments where attenuation is high (due to higher losses). Previous studies on underground transmission using $2.4 \mathrm{GHz}$ showed that due to the high attenuation of soil this frequency band is not feasible [5]. In these environments (i.e., soil) lower frequency bandwidths are preferred due to their lower losses and therefore longer potential range. During this project a WUSN node developed during the research [29] was used as the transmitter with fixed frequencies of $433 \mathrm{MHz}$ and $868 \mathrm{MHz}$. The node was placed at the bottom of the first hole and the hole was backfilled and compacted with the same soil. Handheld RF spectrum analysers were used to detect the signal strength at various distances from the transmitter by burying them or their antenna at the same depth at a fixed horizontal spacing from the transmitter in the other excavated holes. The soil was compacted over the receiver for each location. The RF spectrum analysers were connected by a shielded USB cable to a laptop where the results were logged. Figure 3 shows a schematic of the trials.

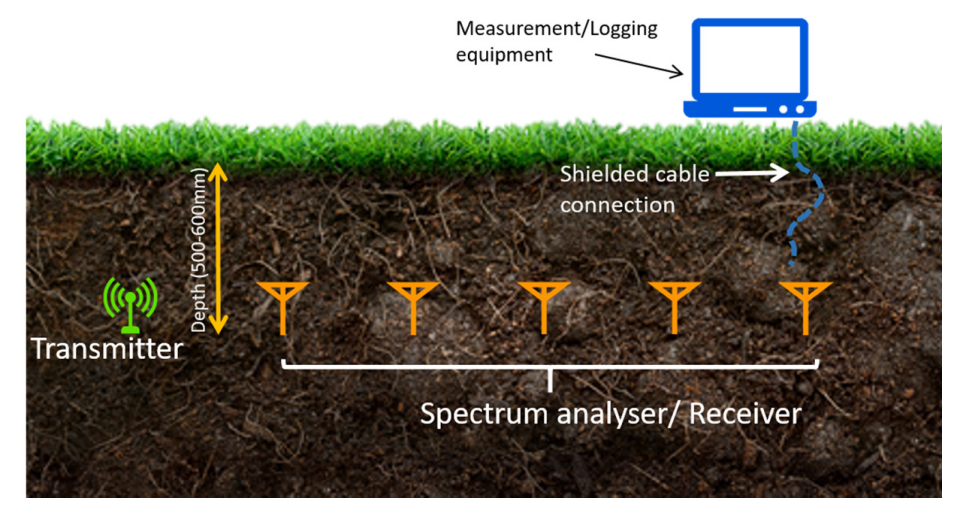

Figure 3. Schematic of the RF trials.

Samples of soil were taken from each of the holes at multiple depths to analyse the characteristics of the soil. The particle size distribution (PSD) and the gravimetric water content of the samples were measured during the laboratory tests [10]. The dry sieving method based on British Standard 1377-2 [30] was used to obtain the PSD of the samples. The conductivity and permittivity of the samples were also measured in the laboratory using Time Domain Reflectometry (TDR) [11,12]. Care was taken during the removal of the soil samples from the ground in order to minimise disturbance to the natural compaction state of the soil.

The main parameters that can potentially negatively affect RF trials are antenna orientation, soil disturbance and instrument characteristics [13]. The high attenuation of EM signals in soil makes the orientation of the antennas a crucial parameter in the tests. For the purposes of these tests, omnidirectional antennas were used and all of the antennas were placed in the soil parallel to each other by ensuring that they were vertical to the horizon before the compaction stage. In addition, based on the parameters used in these tests (i.e., frequency, distance, antennas) and the Fraunhofer distance $\left(d_{f}=\frac{2 D^{2}}{\lambda}\right)$ the measurements are considered to be in the far field region $\left(>d_{f}\right)$ [31]. Any disturbance of the soil will alter its dielectric properties. As the soil is excavated from the holes, irrespective of the excavation method, it will lose its original structure and density. Therefore, in order to ensure repeatability and reliability of the results it was very important that the excavated soil from each hole was compacted back on top of the transmitter/receiver to restore the soil to its original condition as much as possible. In addition, it was also important that the excavation process was carried out in multiple stages to avoid mixing different layers of soil during the backfilling stage. Different equipment can have different performance characteristics; i.e., the antennas and spectrum analysers can have different gains and noise floors. Therefore, to avoid variances in the performance characteristics of the equipment negatively affecting the measurements, the same equipment was used in all of the tests. Measurements of signal intensity were repeated 5 times to investigate repeatability and reliability of the measurements. 


\section{Results and Discussion}

The soil from each of the locations was characterised in the laboratory using British Standard 1377-2 [30]. The results from the soil characterisation tests for all of the locations (A, B and C) are presented in Table 1.

Table 1. Soil characteristic of location A, B and C (GWC: gravimetric water content, $\varepsilon^{\prime}$ real part of the permittivity, $\varepsilon^{\prime \prime}$ imaginary part of the permittivity, $\sigma_{\mathrm{DC}}$ bulk conductivity).

\begin{tabular}{cccccc}
\hline Location & Classification * & GWC & $\varepsilon^{\prime}\left(\right.$ TDR)/ $\varepsilon^{\prime}$ (Peplinski) & $\varepsilon^{\prime \prime}\left(\right.$ TDR)/ $\varepsilon^{\prime \prime}($ Peplinski) & $\sigma_{\text {DC }}(\mathbf{m S} / \mathbf{m})$ \\
\hline A & Gravelly SAND & $12.97 \%$ & $6.53 / 7.14$ & $1.88 / 1.31$ & 2.32 \\
B & Gravelly SAND & $17.02 \%$ & $10.21 / 11.78$ & $1.42 / 1.96$ & 3.74 \\
C & Clayey Silt & $41.72 \%$ & $27.42 / 28.66$ & $5.93 / 6.74$ & 61.23 \\
\hline \multicolumn{5}{c}{ * based on the PSD for each soil. }
\end{tabular}

Table 1 shows a large difference between the soil types and conditions at the locations used for the trials. This helps to investigate the performance of the proposed method in a wide range of input parameters. As can be seen from Table 1, the soil from location $C$ had a significantly higher water content compared to locations $\mathrm{A}$ and $\mathrm{B}$. The soil from location $\mathrm{C}$ also had a considerably higher conductivity compared to the other two locations. Figures 4-6 illustrate the measured attenuation of the signal at locations $\mathrm{A}, \mathrm{B}$ and $\mathrm{C}$ respectively. At location $\mathrm{A}$, it was only possible to record two attenuation measurements; one at $0.2 \mathrm{~m}$ and one at $0.4 \mathrm{~m}$ (depth) from the transmitter. This was due to the practical limitations (i.e., equipment and location) during tests carried out at location $\mathrm{A}$. The methodology for the tests was refined for locations B and C. At location B, five transmitter receiver distances were measured; and at position $C$, attenuation over three distances were measured as seen in Figures 4-6. The predicted values for the RF attenuation for each of the soils were calculated using the CRIM-Fresnel, conventional Modified-Friis and the alternative Modified-Friis (i.e., using TDR measurements) models, and are also displayed in Figures 4-6 for comparison. The error bars in these figures represent the maximum and minimum reading at each point.

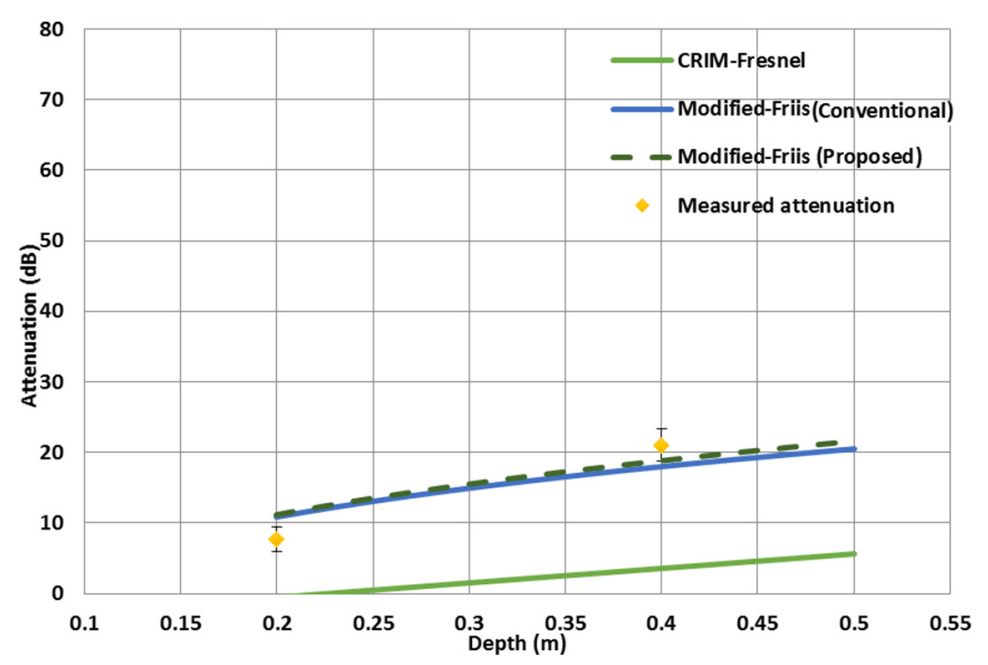

Figure 4. Comparison of the measured attenuation with the propagation models (location A). 


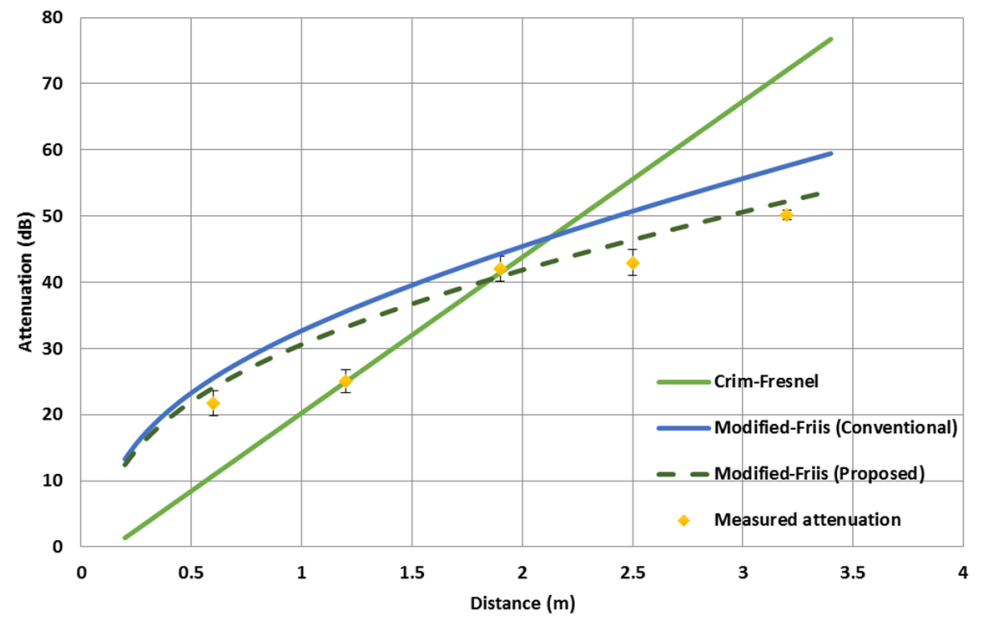

Figure 5. Comparison of the measured attenuation with the propagation models (location B).

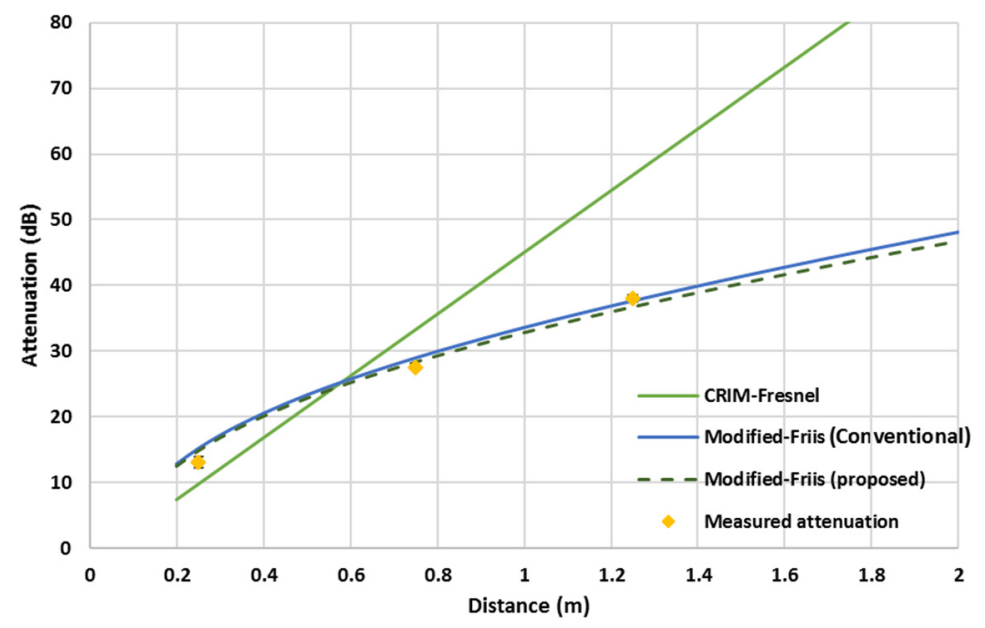

Figure 6. Comparison of the measured attenuation with the propagation models (location C).

As can be seen from the results at all of the locations (Figures 4-6) the Modified-Friis model, using both the conventional and the alternative TDR methods of permittivity estimation, provided a better approximation for the attenuation of the signal in soil compared to the CRIM-Fresnel method. The Root Means Squared Error (RMSE) of these models was calculated in order to compare their 'performance' in predicting the RF attenuation with distance from the transmitter over the distance measured (see Table 2).

Table 2. RMSE for the different models.

\begin{tabular}{cccc}
\hline \multirow{2}{*}{ Location } & \multicolumn{3}{c}{ RMSE } \\
\cline { 2 - 4 } & CRIM-Fresnel & Modified-Friis (Conventional) & New Modified-Friis \\
\hline A & 13.59 & 3.06 & 2.85 \\
B & 12.31 & 7.00 & 4.19 \\
C & 11.58 & 1.47 & 1.36 \\
\hline
\end{tabular}

As shown in Figure 4 at location A, the CRIM-Fresnel model under-estimates the attenuation of the signal (RMSE = 13.59). The conventional Modified-Friis model provided a significantly better approximation (RMSE $=3.06$ ) compared to the CRIM-Fresnel model. However, the estimation of attenuation based on the Modified-Friis model using values obtained from TDR provided the best 
estimation (RMSE = 2.85) compared to the other two methods. As can be seen from Figure 5, and similar to location A, the CRIM-Fresnel model failed to provide an acceptable estimation for the attenuation of the signal at location B (RMSE = 12.31). Figure 5 also shows that both the Modified-Friis estimation models (conventional and TDR based) provided a better estimation for the attenuation of the signal compared to the CRIM-Fresnel model. However, similar to location A, the TDR based Modified-Friis model performed better $($ RMSE $=4.19)$ than the conventional Modified-Friis model $($ RMSE $=7.00)$ at predicting the RF attenuation over the distances measured. Finally, from Figure 6 it can be seen that the performances of the estimation models at location $C$ were very similar to the other two locations despite the considerable difference between the soil type and condition at this location compared to locations A and B. At this location, the CRIM-Fresnel model again provided the least accurate estimation of attenuation (RMSE $=11.58$ ). Both the models based on the Modified-Friis approach were very similar in their estimations. However, similar to locations A and B, the Modified-Friis method with the permittivity estimated from TDR provided a better approximation (RMSE $=1.36$ ) than the conventional Modified-Friis model (RMSE $=1.47$ ), which uses the Peplinski mixing model to estimate permittivity. It should be noted that, although none of the models accurately predict all the measured attenuation values, the Modified-Friis method using permittivity values based on TDR measurements performed equally well, if not better, than the Modified-Friis method using the Peplinski approach to determine the permittivity values. This novel approach will make a significant impact on the community, as the soil does not have to be brought back to the laboratory for assessment, and field-based TDR measurements can be used.

\section{Conclusions}

A large proportion of the infrastructure that our everyday life is dependent on is buried under the ground. Similar to any other infrastructure, buried infrastructure ages and degrades over its lifetime. This makes continuous monitoring of these assets a very important aspect of buried infrastructure management. WSNs have commonly been used for monitoring of above-ground assets due to their scalability and ease of development. However, due to significantly higher attenuation of wireless signals in soil as compared to air, the wireless communication aspect of WUSNs is extremely challenging. In addition, soil is a very complex and dynamic medium which makes accurate estimation of signal attenuation difficult. Therefore, a robust, reliable and easy-to-use method for approximation of the attenuation in soil can hugely benefit the development and deployment of WUSNs. In addition, such a method will provide valuable information for other asset monitoring techniques that rely on propagation of electromagnetic signals in soil (e.g., GPR surveys).

In this paper, the RF attenuation was measured at three field locations with different soil conditions, and these results were then compared to the RF attenuation calculated for these soils using the two main attenuation prediction models (i.e., the Modified-Friis and CRIM-Fresnel models). It has been demonstrated that, for each of the three soils at the locations tested, the Modified-Friis model for estimating signal attenuation more closely fitted the field data compared to the CRIM-Fresnel model. Although only three soil types were used, they represented a wide range of soil conditions. However, confidence in the findings could be increased by adding more test data.

In addition, an alternative method for determining the soil permittivity for use in the Modified-Friis model was also investigated in this paper. This technique estimates the effective frequency of the Time Domain Reflectometry (TDR) signal in the soil by using the rise time of the signal at the end of the TDR probe, and uses this effective frequency to calculate the complex permittivity. The use of the steady state reflection coefficient was proposed as a more robust way to determine the upper intercept of the rise time over the conventional method for rise time extraction. Using this steady state reflection coefficient significantly improves the reliability and robustness of this process by eliminating the inaccuracies caused by the common end reflections in the TDR waveform. The use of the TDR measured permittivity is suggested to improve the accuracy of the Modified-Friis model as it is based on measurements of the dielectric parameters of the soil at the 
WUSN location. In addition, it removes the need for laboratory-based tests for calculating the dielectric parameters, which makes it more suitable for field use (e.g., prior to conducting GPR surveys). The performance of this alternative Modified-Friis method for estimating the RF attenuation was analysed by comparing the predicted attenuation with the measured field data, and also with the CRIM-Fresnel and conventional Modified-Friis models. These comparisons showed that in all three field trial locations the Modified-Friis model with the complex permittivity extracted from TDR data of the soils provided a better estimation of the RF attenuation over the distances measured. These results are extremely promising, and provide evidence for the proposed alternative TDR-based method for estimating the RF attenuation in soil and even show that this method results in estimations that are more accurate.

Acknowledgments: The authors gratefully acknowledge the support by the University of Birmingham, UK and Water Industry Research (UKWIR) who funded different parts of this project.

Author Contributions: Ali Sadeghioon conducted the research and trials. David Chapman, Nicole Metje and Carl Anthony supervised the research and added their intellectual property and experience into the system, particularly the field trials. All the authors helped write the paper.

Conflicts of Interest: The authors declare no conflict of interest.

\section{Abbreviations}

The following abbreviations are used in this manuscript:

$\begin{array}{ll}\text { RF } & \text { Radio Frequency } \\ \text { TDR } & \text { Time Domain Reflectometry } \\ \text { BEC } & \text { Bulk Electrical Conductivity } \\ \text { PSD } & \text { Particle Size Distribution } \\ \text { EM } & \text { Electromagnetic } \\ \text { GPR } & \text { Ground Penetrating Radar } \\ \text { WSN } & \text { Wireless Sensor Networks } \\ \text { WUSN } & \text { Wireless Underground Sensor Networks }\end{array}$

\section{References}

1. Balachander, D.; Rao, T.R.; Mahesh, G. RF Propagation Investigations in Agricultural Fields and Gardens for Wireless Sensor Communications. In Proceedings of the 2013 IEEE Conference on Information and Communication Technologies, Tamil Nadu, India, 11-12 April 2013; pp. 755-759.

2. Hancke, G. Industrial wireless sensor networks: A selection of challenging applications. In Proceedings of the 2012 6th European Conference on Antennas and Propagation (EUCAP), Prague, Czech, 26-30 March 2012; pp. 64-68.

3. Akyildiz, I.F.; Stuntebeck, E.P. Wireless underground sensor networks: Research challenges. Ad Hoc Netw. 2006, 4, 669-686. [CrossRef]

4. The National Joint Utiities Group (NJUG). NJUG Guidelines on the Positioning and Colour Coding of Underground Utilities' Apparatus; NJUG: London, UK, 2007.

5. Silva, A.R.; Vuran, M.C. Empirical Evaluation of Wireless Underground-to-Underground Communication in Wireless Underground Sensor Networks; Springer: Berlin/Heidelberg, Germany, 2009; Volume 5516.

6. Kurt, S.; Tavli, B. Path-Loss Modeling for Wireless Sensor Networks. IEEE Antennas Propag. Mag. 2017, 59, 18-37. [CrossRef]

7. Alsayyari, A.; Kostanic, I.; Otero, C.; Mohammed, A.; Kusay, R. An Empirical Path Loss Model for Wireless Sensor Network Deployment in a Sand Terrain Environment. In Proceedings of the 2014 IEEE World Forum on Internet of Things (WF-IoT), Seoul, Korea, 6-8 March 2014; pp. 218-223.

8. Aisayyari, A.; Kostanic, I.; Otero, C.E. An Empirical Path Loss Model for Wireless Sensor Network Deployment in an Artificial Turf Environment. In Proceedings of the 11th IEEE International Conference on Networking, Sensing and Control, Miami, FL, USA, 7-9 April 2014; pp. 637-642.

9. Silva, A.R. Channel Characterization for Wireless Underground Sensor Networks. Master's Thesis, University of Nebraska, Lincoln, NE, USA, 2010. 
10. Sadeghioon, A.M. Design and Development of Wireless Underground Sensor Networks for Pipeline Monitoring. Ph.D. Thesis, School of mechanical enginrring, University of Birmingham, Birmingham, UK, 2015.

11. Curioni, G. Investigating the Seasonal Variability of Electromagnetic Soil Properties Using Field Monitoring Data from Time-Domain Reflectometry Probes. Ph.D. Thesis, University of Birmingham, Birmingham, UK, 2013.

12. Topp, G.; Zegelin, S.; White, I. Impacts of the real and imaginary components of relative permittivity on time domain reflectometry measurements in soils. Soil Sci. Soc. Am. 2000, 1252, 1244-1252. [CrossRef]

13. Silva, A.R.; Vuran, M.C. Development of a Testbed for Wireless Underground Sensor Networks. EURASIP J. Wirel. Commun. Netw. 2010, 1-14. [CrossRef]

14. Yu, X.; Wu, P.; Han, W.; Zhang, Z. Overview of wireless underground sensor networks for agriculture. African J. Biotechnol. 2012, 11, 3942-3948. [CrossRef]

15. Yoon, S. Wireless Signal Networks: A Proof of Concept for Subsurface Characterization and A System Design with Reconfigurable Radio. Ph.D. Thesis, Lehigh University, Bethlehem, PA, USA, 2013.

16. Van-Dam, R.L.; Borchers, B.; Hendrickx, J.M.H. Methods for prediction of soil dielectric properties: A review Remke. Def. Secur. 2005, 5794, 188-197.

17. Peplinski, N.; Ulaby, F.T.; Dobson, M.C. Dielectric properties of soils in the 0.3-1.3-GHz range. IEEE Trans. Geosci. Remote Sens. 1995, 33, 803-807. [CrossRef]

18. Peplinski, N.; Ulaby, F.T.; Dobson, M.C. Corrections to “Dielectric Properties of Soils in the 0.3-1.3-GHz Range". IEEE Trans. Geosci. Remote Sens. 1995, 33, 1340. [CrossRef]

19. Mironov, V.; Dobson, M. Generalized refractive mixing dielectric model for moist soils. Geosci. Remote Sens. IEEE Trans. 2004, 42, 3556-3558. [CrossRef]

20. Robinson, D.A.; Jones, S.B.; Wraith, J.M.; Or, D.; Friedman, S.P. A Review of Advances in Dielectric and Electrical Conductivity Measurement in Soils Using Time Domain Reflectometry. Vadose Zone J. 2003, 2, 444-475. [CrossRef]

21. Chaamwe, N.; Liu, W.; Jiang, H. Wave Propagation Communication Models for Wireless Underground Sensor Networks. In Proceedings of the 12th IEEE International Conference on Communication Technology (ICCT), Nanjing, China, 11-14 November 2010; pp. 9-12.

22. Sahota, H.; Kumar, R. Network based sensor localization in multi-media application of precision agriculture Part 1: Received signal strength. In Proceedings of the 11th IEEE International Conference on Networking, Sensing and Control, Miami, FL, USA, 7-9 April 2014; pp. 191-196.

23. Bogena, H.R.; Huisman, J.A.; Meier, H.; Rosenbaum, U.; Weuthen, A. Hybrid Wireless Underground Sensor Networks: Quantification of Signal Attenuation in Soil. Vadose Zone J. 2009, 8, 755-761. [CrossRef]

24. Li, L.; Vuran, M.; Akyildiz, I. Characteristics of underground channel for wireless underground sensor networks. In Proceedings of the Sixth Annual Mediterranean Ad Hoc Networking WorkShop, Corfu, Greece, 12-15 June 2007; pp. 92-99.

25. Vuran, M.C.; Silva, A.R. Communication Through Soil in Wireless Underground Sensor Networks-Theory and Practice. In Sensor Networks, Signals and Communication Technology; Ferrari, G., Ed.; Springer: Berlin, Germany, 2009; pp. 309-347.

26. Friis, H. A note on a simple transmission formula. Proc. IRE 1946, 34, 254-256. [CrossRef]

27. Dane, J.H.; Topp, C. Methods of Soil Analysis: Part 4; Soil Science Society of America, Inc.: Madison, WI, USA, 2002.

28. Evett, S.R.; Parkin, G.W. Advances in Soil Water Content Sensing. Vadose Zone J. 2005, 4, 986. [CrossRef]

29. Sadeghioon, A.M.; Metje, N.; Chapman, D.; Anthony, C. SmartPipes: Smart Wireless Sensor Networks for Leak Detection in Water Pipelines. J. Sens. Actuator Netw. 2014, 3, 64-78. [CrossRef]

30. British Standards Institution (BSI). BS 1377-2 Methods of Test for Soils for Civil Engineering Purposes: Part 2: Classification Tests; BSI: London, UK, 1990.

31. Rappaport, T.S. Wireless Communications: Principles and Practice, 2nd ed.; Prentice Hall: Englewood Cliffs, NJ, USA, 2001.

(C) 2017 by the authors. Licensee MDPI, Basel, Switzerland. This article is an open access article distributed under the terms and conditions of the Creative Commons Attribution (CC BY) license (http:// creativecommons.org/licenses/by/4.0/). 